\title{
Nutrición vía oral precoz en pancreatitis aguda. Estudio de cohorte
}

\author{
Héctor Losada M..$^{1,2,3}$, Andrés Troncoso T. ${ }^{1,2}$, Sonia Curitol S. ${ }^{1,2}$, \\ Felipe Sanhueza V. ${ }^{1,2}$ y Jorge Silva A. ${ }^{1,2,3}$.
}

'Departamento de Cirugía, Universidad de La Frontera. ¿Equipo de Cirugía Biliopancreática, Hospital Regional de Temuco.

${ }^{3}$ Equipo de Cirugía Biliopancreática, Departamento de Cirugía Clínica Alemana de Temuco. Temuco, Chile

Financiado por proyecto DIDUFRO No $17-0025$ Publicación: este estudio se presentó en el III Congreso Latinoamericano de Cirugía Hepatopancreática y bilia Chile 2017 (septiembre, Viña del Mar) y en el $90^{\circ}$ Congreso Chileno e Internacional de Cirugía 2017 (noviembre, Pucón)

Recibido 2020-08-06 y aceptado 2020-10-26

Correspondencia a: Dr. Héctor Losada M. hector.losada@ufrontera.c

\section{Early oral nutrition in acute pancreatitis. Cohort study}

Aim: To determinate if the tolerance to early oral nutrition (NVOP) decreases hospital stay and is not associated with greater reactivation of acute pancreatitis (AP) or worst prognosis compared to patients that did not tolerate NVOP protocol. Materials and Method: A prospective cohort of patients with AP who NVOP protocol was applied then we evaluated protocol tolerance, feedback time, hospital stay, AP reactivation, presence of local complications, and need for bed in critical care unit. The results were compared between patients who tolerate and did not tolerate this protocol. Results: 65 patients were included, $69.2 \%$ female. $49.3 \%$ presented severe acute pancreatitis (PAG) and $90.8 \%$ tolerated the NVOP protocol, with a mean hospital stay of $13.3 \pm 5$ days vs. $19 \pm 8.2$ days in those who did not tolerate it $(\mathrm{p}=0,0177)$. There was reactivation of AP in $1(1.5 \%)$ patient without relation to the protocol. Nine patients were excluded, because they did not achieve criteria to start or maintain protocol. Discussion: The AP is a frequent reason for consultation in the emergency services of our country, so achieving standardization in nutritional therapy is of great importance to reduce morbidity and mortality. Our results are comparable with international studies supporting early enteral nutrition. Conclusion: The tolerance to NVOP as treatment of AP it is associated with shorter hospital stay, and is not related to a higher rate of reactivation of AP, local complications, or increased mortality compared to the patients who did not tolerate this protocol.

Key words: pancreatitis; nutrition therapy; oral nutrition; cohort study.

\section{Resumen}

Objetivo: Determinar si la nutrición vía oral precoz (NVOP) disminuye la estancia hospitalaria y no se asocia a mayor reactivación de pancreatitis aguda (PA), ni a deterioro del pronóstico. Materiales y Método: Cohorte prospectiva de pacientes con PA, a los cuales se les aplicó un protocolo de NVOP y se evaluó tolerancia, tiempo de realimentación, estancia hospitalaria, reactivación de PA, complicaciones locales y necesidad de cama en unidad de cuidado crítico. Comparamos resultados entre pacientes que toleraron y no toleraron dicho protocolo. Resultados: Incluimos 65 pacientes, 69,2\% de género femenino. El 49,3\% presentó pancreatitis aguda grave (PAG) y $90,8 \%$ toleró protocolo de NVOP, con promedio de estancia hospitalaria de 13,3 \pm 5 días $v s .19 \pm 8,2$ días en quienes no toleraron $(\mathrm{p}=0,0177)$. Hubo reactivación de PA en $1(1,5 \%)$ paciente sin tener relación con el protocolo. No hubo necesidad de cama UTI/UCI, ni mortalidad. Nueve pacientes fueron excluidos por no cumplir criterios para iniciar o mantener protocolo. Discusión: La PA es motivo frecuente de consulta en los servicios de urgencia de nuestro país, por lo que lograr una estandarización en la terapia nutricional resulta de mucha importancia para disminuir la morbimortalidad. Nuestros resultados son comparables con estudios internacionales que apoyan la nutrición enteral precoz. Conclusión: La tolerancia a la NVOP en el tratamiento de la PA, se asocia a menor estancia hospitalaria, menor tasa de reactivación de PA, menos complicaciones locales y sin aumentar la mortalidad, en relación con los que no toleran la NVOP.

Palabras clave: pancreatitis; terapia nutricional; estudio de cohorte; nutrición oral. 


\section{Introducción}

La pancreatitis aguda (PA) representa una patología con una prevalencia en los EE.UU. de más de 200.000 casos por año, y en Chile alrededor de los $3.000^{1}$. Para nuestro centro asistencial, Hospital Dr. Hernán Henríquez Aravena de Temuco (Chile), es una patología frecuente debido a la alta prevalencia de colelitiasis en la novena región, estimándose un promedio de 200 pacientes al año ${ }^{2}$.

La alimentación en la PA representa un pilar clave como parte del tratamiento. Se han propuesto distintos métodos para reiniciar la alimentación en pacientes con esta patología: la vía parenteral y la enteral (la más aceptada), y se recomienda iniciar de forma precoz ya sea vía oral, mediante sonda nasogástrica o sonda nasoyeyunal, pues se ha demostrado que se asocia a buenos resultados en pacientes con PA leve y presenta buenos resultados en PAG según algunos trabajos, sin embargo, aún no hay evidencia categórica sobre esta última ${ }^{3-5}$.

La hipótesis de nuestra investigación es que la tolerancia a la Nutrición Vía Oral Precoz (NVOP) en pacientes con PA disminuye la estancia hospitalaria y no se asocia a un aumento en la reactivación ni un deterioro en el pronóstico en relación con los pacientes que no la toleran. El objetivo de nuestro trabajo es precisar si la tolerancia a la NVOP determina una disminución de la estancia hospitalaria y si se asocia a menor tasa de reactivación de PA en relación a los que no la toleran.

\section{Materiales y Método}

\section{Diseño}

Estudio de cohorte prospectiva.

\section{Población}

Pacientes con PA hospitalizados en el Hospital Dr. Hernán Henríquez Aravena de la ciudad de Temuco entre el 01 de abril de 2016 y el 31 de enero de 2017, manejados por el equipo de cirugía hepatobiliopancreática de este centro. Se excluyeron los pacientes que requirieron ventilación mecánica, que presentaron íleo gástrico o sospecha de obstrucción intestinal.

\section{Maniobra}

A los pacientes diagnosticados con PA (niveles séricos de amilasa y/o lipasa 3 veces sobre el valor normal en su límite superior) que tuvieron ausencia de dolor abdominal espontáneo (independiente del esquema de analgesia), náuseas o emesis y no pre- sentaron signos de irritación peritoneal al examen físico, se les midió nuevamente el nivel sérico de enzimas pancreáticas. Si el valor presentaba una disminución de más del $50 \%$ del valor previo (o con valor normal), se iniciaba el protocolo de NVOP. Este se inició independientemente del valor de proteína $\mathrm{C}$ reactiva (PCR), leucocitos u otras variables (Figura 1).

Para el cálculo de gravedad de PA se utilizó el score de APACHE II y la medición PCR; considerando como PAG cuando PCR $\geq$ a $150 \mathrm{mg} / \mathrm{dl} \mathrm{o}$ APACHE II $\geq 8^{6}$. Se utilizaron éstos como criterios de gravedad ya que, según un estudio de cohorte de nuestro centro, tienen mayor asociación con la mortalidad y con la necesidad de camas en UPC ${ }^{2}$.

El protocolo se inició con régimen hídrico el primer día; el segundo día régimen líquido y el tercer día régimen liviano (bajo en grasas). El paciente pudo tener una progresión lenta del esquema si presentó algún síntoma abdominal, pero podía permanecer máximo $48 \mathrm{~h}$ sin progresión al régimen más complejo. Si toleraba este esquema se consideraba exitoso el protocolo de NVOP.

Si el paciente persistió con régimen hídrico o líquido por más de $48 \mathrm{~h}$ por dolor abdominal y/o náuseas y/o emesis, se consideró fracaso del protocolo de NVOP.

En este estudio no se consideró como variable el estado nutricional de los pacientes, requerimientos calórico-proteicos, grado de catabolismo ni se cuantificó el aporte nutricional vía oral.

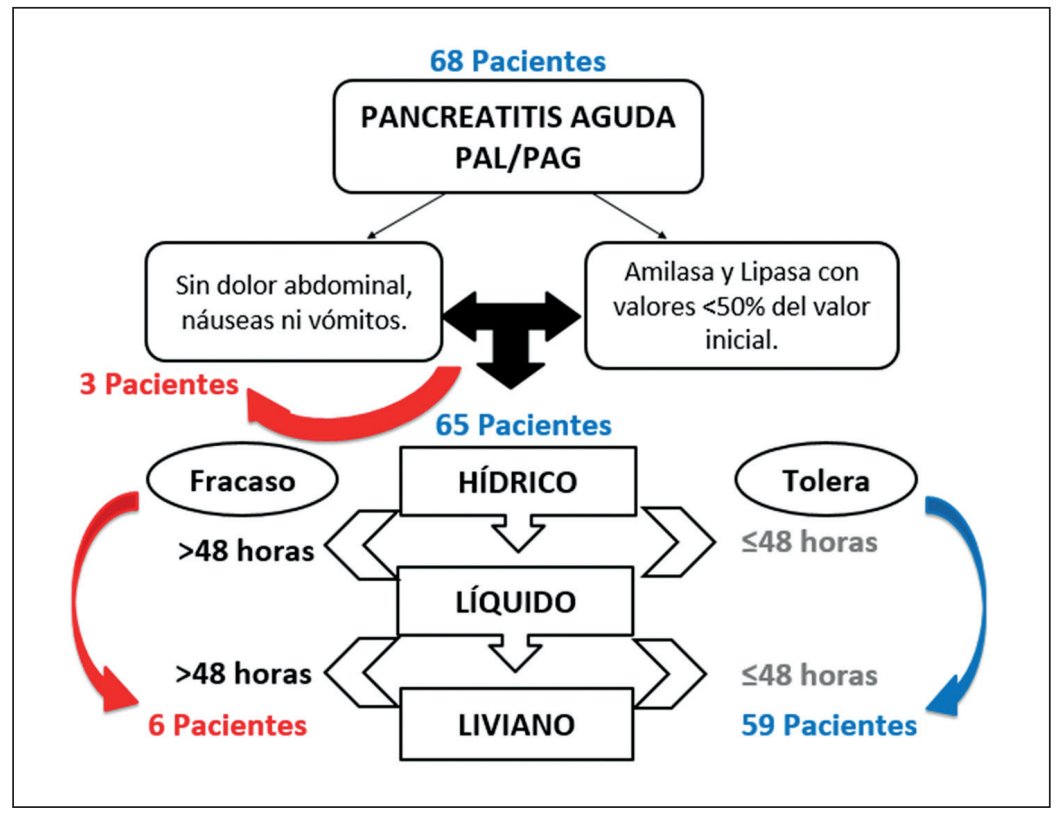

Figura 1. Flujograma de pacientes incluidos y excluidos. 


\section{Variables de desenlace}

1. Intolerancia a NVOP: necesidad de suspender la vía oral por dolor abdominal, emesis, náuseas o diarrea. Se midió como variable dicotómica.

2. Complicaciones infecciosas locales: medida en términos de presencia de complicaciones locales (necrosis pancreática o peripancreática; colección asociada a necrosis, pseudoquiste).

3. Requerimiento de ingreso a Unidad de Paciente Crítico (UPC): Medida como variable dicotómica (presente o ausente), cuando la causa de ingreso fue disfunción orgánica o sepsis.

4. Reactivación de PA: Dolor abdominal y aumento de enzimas pancreáticas. Si el valor previo es normal se consideró como significativo un aumento de 3 veces el valor normal alto. Si el valor previo no es normal, se consideró como significativo cualquier aumento en las cifras.

5. Estancia hospitalaria: días totales de hospitalización. Variable continua.

\section{Cálculo del tamaño de la muestra}

Para el cálculo del tamaño de la muestra se definió como variable desenlace la estancia hospitalaria. En un reporte de nuestro grupo ${ }^{4}$, se obtuvo que la mediana de hospitalización en pacientes con PA fue de 14 días. En este estudio el promedio fue de $15 \pm 8$ días. Se proyectó que con el protocolo de NVOP se podía disminuir el promedio de estancia hospitalaria a 9 días. Con un nivel de significancia de $95 \%$ y un poder de $80 \%$ se obtuvo un tamaño de la muestra de 64 pacientes.

\section{Herramientas estadísticas}

Se utilizó herramientas de estadística descriptiva con medidas de tendencia central y de dispersión; herramientas de estadística analítica con el $\chi^{2}$ o test exacto de Fisher para variables dicotómicas y t-test para variables continuas una vez hecho el análisis de varianzas.

La base de datos para el estudio fue confeccionada en Excel $^{\circledR}$ y el análisis se realizó en el programa Stata $^{\circledR} 12.0$.

\section{Aspectos éticos}

El protocolo fue aprobado por el comité de ética científica del Servicio de Salud Araucanía Sur (Chile) y por el Grupo de Investigación del Departamento de Cirugía, Traumatología y Anestesiología de la Universidad de la Frontera.

\section{Financiamiento}

El proyecto fue financiado por proyecto DIUFRO $\mathrm{N}^{\circ} 17-0025$.

\section{Resultados}

La cohorte está constituida por 65 pacientes, 45 $(69,2 \%)$ de género femenino y $20(30,7 \%)$ de género masculino, con una edad promedio de 49,1 $\pm 19,5$ años (Tabla 1).

En cuanto a la gravedad de la cohorte global, 32 (49,3\%) pacientes corresponden a PAG (20 con APACHE II mayor o igual a 8 puntos y 12 con PCR mayor o igual a $150 \mathrm{mg} / \mathrm{L})$ y $33(50,7 \%)$ a $\mathrm{PAL}$ (Tabla 1).

Al evaluar la etiología de la PA, en $52(80 \%)$ pacientes fue litiásica y en $13(20 \%)$ no se pudo objetivar origen litiásico (por lo que se consideraron alitiásicas) (Tabla 1). Se realizó colecistectomía en $44(67,6 \%)$ pacientes, ERCP en $7(10,7 \%)$ y 1 rechazó cirugía.

El promedio de APACHE II de ingreso de la cohorte global fue de $6,19 \pm 4,2$ y de PCR fue $69,97 \pm 79,8 \mathrm{mg} / \mathrm{dl}$ y al reevaluar a las $48 \mathrm{~h}$ el APACHE II fue de 5,21 \pm 4,1 y PCR de 116,59 $\pm 108,3$ $\mathrm{mg} / \mathrm{dl}$ (Tabla 2).

La estancia hospitalaria de la cohorte global fue de $13,8 \pm 5,6$ días. No hubo pacientes con necesidad de cama en UPC.

Al evaluar tolerancia al protocolo de NVOP: 59 $(90,8 \%)$ pacientes lo toleraron de forma exitosa y $6(9,2 \%)$ pacientes no lo toleraron por persistencia del dolor y/o presencia de náuseas y vómitos. El tiempo promedio para completar la realimentación (desde régimen hídrico hasta régimen liviano) en el grupo que toleró la NVOP fue de 3,8 $\pm 0,9$ días y en los pacientes que no toleraron la NVOP fue de $11,3 \pm 1,5$ días $(\mathrm{p}=0,00001)$.

En cuanto a la reactivación de la PA, en el grupo que toleró la NVOP se presentó solo $1(1,5 \%)$ caso, y ésta se produjo $48 \mathrm{~h}$ posterior al término del protocolo. En el grupo que no toleró la NVOP no hubo reactivación de $\mathrm{PA}$.

De los $6(9,2 \%)$ pacientes que no toleraron la NVOP ninguno requirió cama en UPC, ni alimentación por vía parenteral. En este grupo se presentó una complicación local, un pseudoquiste pancreático.

Al analizar la gravedad de la PA con la asociación a la tolerancia del protocolo de NVOP, tenemos que $30(50,8 \%)$ pacientes en el grupo que toleró la NVOP cursaron con una PAG y en el grupo que no toleró, $2(33 \%)$ pacientes cursaron con una PAG $(\mathrm{p}=0,672)$ (Tabla 1$)$.

Al analizar el promedio de APACHE II y PCR y su asociación con la tolerancia al protocolo de NVOP, tenemos que en el grupo que toleró el protocolo de NVOP el promedio de APACHE II de 
Tabla 1. Características de la cohorte en relación con tolerancia NVOP

\begin{tabular}{|lcccc|}
\hline Variable & Tolera NVOP & No tolera NVOP & Total & P value \\
Género femenino (\%) & 91,1 & 8,8 & 69,2 & 1,00 \\
\hline Edad (años promedio \pm DS) & $48,86 \pm 19,6$ & $51,5 \pm 20,7$ & $49,1 \pm 19,5$ & 0,756 \\
PAG (\%) & 50,8 & 33,3 & 49,2 & 0,672 \\
Litiásica (\%) & 90,3 & 9,6 & 80 & 1,00 \\
Cohorte total & 59 & 6 & 65 & \\
\hline
\end{tabular}

* $\mathrm{PAG}=$ Pancreatitis aguda grave.

Tabla 2. Indicadores de gravedad en relación con tolerancia NVOP

\begin{tabular}{|lcccc|}
\hline Variable & $\begin{array}{c}\text { Tolera NVOP } \\
\text { (Promedio } \pm \text { DS) }\end{array}$ & $\begin{array}{c}\text { No tolera NVOP } \\
\text { (Promedio } \pm \text { DS) }\end{array}$ & $\begin{array}{c}\text { Total } \\
\text { (Promedio } \pm \text { DS) }\end{array}$ & P value \\
Reactivación PA* & 1 & 0 & 1 & 0,092 \\
\hline APACHE II ingreso (puntos) & $6,17 \pm 4,1$ & $6,33 \pm 5$ & $6,19 \pm 4,2$ & 0,9327 \\
APACHE II 48 h (puntos) & $5,22 \pm 4,3$ & $5,16 \pm 2,4$ & $5,21 \pm 4,1$ & 0,9756 \\
PCR ingreso ( mg/L) & $69,86 \pm 82,2$ & $71,05 \pm 56,8$ & $69,97 \pm 79,8$ & 0,9725 \\
PCR 48 h (mg/L) & $116,04 \pm 103,1$ & $121,9 \pm 162,2$ & $116,59 \pm 108,3$ & 0,9009 \\
Estancia hospitalaria (días) & $13,36 \pm 5$ & $19 \pm 8,2$ & $13,89 \pm 5,6$ & 0,0178 \\
Cohorte total & 59 & 6 & 65 & \\
\hline
\end{tabular}

$* \mathrm{PA}=$ Pancreatitis aguda.

ingreso fue de $6,17 \pm 4,1$ y en el grupo que no toleró fue de $6,33 \pm 5(\mathrm{p}=0,9327)$. El promedio APACHE II a las $48 \mathrm{~h}$ fue de 5,22 \pm 4,3 en el grupo que toleró y $5,16 \pm 2,4$ en el grupo que no toleró $(\mathrm{p}=0,9756)$.

El grupo que toleró el protocolo de NVOP tuvo un nivel promedio de PCR sérica de ingreso de $69,86 \pm 82,2 \mathrm{mg} / \mathrm{dl}$ y el grupo que no toleró tuvo uno de 71,05 $\pm 56,8 \mathrm{mg} / \mathrm{dl}(\mathrm{p}=0,9725)$. El valor promedio de los niveles séricos de PCR al control a las $48 \mathrm{~h}$ fue de $116,04 \pm 103,1 \mathrm{mg} / \mathrm{dl}$ en el grupo que toleró y $121,9 \pm 162,2 \mathrm{mg} / \mathrm{dl}$ en el grupo que no toleró $(\mathrm{p}=0,9009)$ (Tabla 2$)$.

Al analizar la estancia hospitalaria y su asociación con la tolerancia al protocolo de NVOP, tenemos que el grupo que no toleró la NVOP tuvo una estancia hospitalaria de $19 \pm 8,2$ días y el grupo que toleró una estancia hospitalaria de 13,36 \pm 5 días, que fue estadísticamente significativa $(\mathrm{p}=0,0178)$ (Tabla 2).

La cohorte no presentó mortalidad y 9 pacientes fueron excluidos por no cumplir criterios para iniciar o mantener el protocolo.

\section{Discusión}

En esta cohorte de pacientes con PA demostramos que evaluar la tolerancia a la NVOP es un método sencillo, viable y seguro. En este estudio el resultado reflejó que quienes toleran la NVOP presentaron una menor estancia hospitalaria, sin relacionarse con una mayor tasa de reactivación de PA, ni complicaciones locales en comparación con los que no la toleraron. La asociación de la no tolerancia al protocolo NVOP y mayor mortalidad se puede deber a la mayor gravedad de los pacientes, de acuerdo con los parámetros utilizados (APACHE II y PCR) e incluso la no tolerancia a la vía oral precoz pudiera ser, de manera independiente, un factor pronóstico de complicaciones (mayor requerimiento de camas UPC) y mortalidad.

La PA sigue siendo un motivo frecuente de consulta en los servicios de urgencia de nuestro país, así como de derivación desde hospitales menos complejos hacia centros de mayor complejidad como el Hospital Hernán Henríquez Aravena de 
Temuco. Es por esto que lograr una estandarización en la terapia de estos pacientes resulta de mucha importancia para disminuir la morbimortalidad.

Como la nutrición juega un rol fundamental en el tratamiento de la pancreatitis ${ }^{7}$, es de gran interés evaluar cómo se comportan los pacientes frente al protocolo de NVOP, compararlo con la evidencia científica disponible y así poder aplicar los resultados de manera óptima en los pacientes, desde su ingreso al servicio de urgencia de nuestro centro asistencial.

La nutrición parenteral (NP) fue históricamente recomendada para los pacientes con PA. Ofrece el beneficio de proporcionar nutrientes exógenos para mantener la masa corporal magra y evitar el íleo adinámico $^{5}$. El uso y abuso de las indicaciones de la nutrición parenteral total ha llevado a cuestionar su real utilidad. La prolongación del reposo digestivo se basa en la prevención de la reactivación de la enfermedad, sin embargo, la nutrición parenteral total como único soporte nutricional se ha asociado a atrofia intestinal y disminución en la función inmunológica de este órgano ${ }^{8-10}$. El uso de la vía enteral ya sea por sonda nasogástrica o vía oral, mantiene la integridad fisiológica intestinal, contribuyendo a disminuir el riesgo de translocación bacteriana, posible punto de partida endógeno de complicaciones sépticas ${ }^{11}$. Una revisión sistemática de 2018 que incluyó 1.424 pacientes concluyó que la nutrición enteral temprana (dentro de las $48 \mathrm{~h}$ posteriores al ingreso) disminuyó significativamente el riesgo de falla orgánica múltiple (FOM), intervención quirúrgica, infecciones sistémicas y complicaciones sépticas locales ${ }^{12}$. En el mismo año, otro metaanálisis que revisó 11 ensayos clínicos aleatorizados (ECA) y un total de 562 pacientes presenta hallazgos similares ${ }^{13}$.

Al igual que en otras series nacionales e internacionales ${ }^{14-17}$, en nuestra cohorte el predominio de pacientes corresponde al sexo femenino. Llama la atención que en esta cohorte no se produce distribución 4:1 entre PAL y PAG descrita en la literatura, sino que se obtiene una proporción más cercana de $1: 1$, tendencia que se mantiene en ambos grupos al separarlos por género. En cuanto a la etiología se mantiene lo descrito en la literatura ${ }^{18}$, de que es mayoritariamente de origen biliar (litiásico).

Algunos ensayos clínicos y metaanálisis dan cuenta que la realimentación precoz, sobre todo antes de las $48 \mathrm{~h}$ se asocia a una disminución en el riesgo de FOM, complicaciones infecciosas pancreáticas y mortalidad ${ }^{19-21}$ y es por esto que las sociedades de nutrición tanto americanas como europeas recomiendan el uso precoz de la alimentación, inclusive con sonda nasoenteral, si es que no se han realimentado al séptimo día ${ }^{22,23}$. Estos potenciales beneficios se replican en nuestro estudio, en donde no encontramos relación directa entre la NVOP y la necesidad de cama en UPC, la reactivación de PA ni aumento en la mortalidad.

En nuestro estudio optamos por la alimentación vía oral para todos nuestros pacientes, dejando a un lado nuestro protocolo anterior ${ }^{6}$, de utilizar alimentación por sonda nasoenteral en el grupo de PAG; esto porque es mejor tolerada, tal como se planteó en un ensayo clínico randomizado ${ }^{20}$, donde se sostiene que es un procedimiento seguro, eficaz y viable. En este mismo contexto el uso de sonda nasoenteral se asocia a molestias, mordeduras o esofagitis y con frecuencia se desprende o se obstruye, lo que hace necesario reemplazar esta última ${ }^{24}$. En nuestra cohorte no apreciamos diferencias al usar la vía oral, tanto en pacientes con PAL como PAG.

La variable de desenlace en este estudio es la estancia hospitalaria, la cual mostró disminución de $19 \pm 8,2$ días en el grupo que no toleró la NVOP a $13,3 \pm 5$ días en el grupo que toleró la NVOP. Esta variable puede estar alterada, ya que en nuestro equipo decidimos no dar de alta al paciente hasta no resolver la patología litiásica que originó el cuadro de PA. Debido al gran número de casos con patología oncológica biliopancreática de nuestro equipo, es posible que se prolongue la resolución de la patología litiásica. Sin embargo, un hecho relevante es el tiempo para completar la realimentación: $3,8 \pm 0,9$ días en los pacientes que toleraron el protocolo de NVOP y 11,3 $\pm 1,5$ días en los pacientes que no toleraron el protocolo.

Cabe destacar que del total de la cohorte (65 pacientes), el 90,7\% toleró con éxito el protocolo de NVOP, siendo el $46 \%$ de estos pacientes catalogados como PAG, dicho de otra manera, el 93,75\% de los pacientes con PAG toleró con éxito el protocolo de alimentación; por lo tanto, la gravedad de la pancreatitis no fue un impedimento para realimentar a estos pacientes de manera precoz.

\section{Conclusión}

En nuestra cohorte de pacientes con PA la tolerancia al protocolo de NVOP, está asociada a una menor estancia hospitalaria y no se relaciona a una mayor tasa de reactivación, complicaciones locales, ni necesidad de cama en UPC en relación con los que no toleraron protocolo. 


\section{Responsabilidades éticas}

Protección de personas y animales. Los autores declaran que para esta investigación no se han realizado experimentos en seres humanos ni en animales.
Confidencialidad de los datos. Los autores declaran que en este artículo no aparecen datos de pacientes.

Conflictos de interés: no hay.

\section{Bibliografía}

1. Gompertz M, Fernández L, Lara I, Miranda J, Mancilla C, Berger Z. Índice clínico de gravedad en pancreatitis aguda: BISAP ("Bedside Index for Severity in Acute Pancreatitis"): Dos años de experiencia en el Hospital Clínico Universidad de Chile. Rev Med Chile 2012;140:977-83.

2. Losada H, Troncoso A, Silva J, Burgos San Juan L, Acencio L, Arias O. APACHE II, proteína $\mathrm{C}$ reactiva y score de Marshall en pancreatitis aguda. Asociación con el ingreso a unidad de paciente crítico. Estudio de cohorte. Congreso Chileno de Cirujanos, Antofagasta 2014

3. Oláh A, Romics Jr L. Enteral nutrition in acute pancreatitis: A review of the current evidence. World Journal of Gastroenterology 2014;20:16123-31.

4. Lipovestky F, Ramos A, Cueto G, Tonelli C, Guimaraens P, Reina R, et al. Pancreatitis aguda. Su manejo en Cuidados Intensivos. Rev Arg de Ter Int. 2016;33:1-13.

5. Li W, Liu J, Zhao S, Li J. Safety and efficacy of total parenteral nutrition versus total enteral nutrition for patients with severe acute pancreatitis: a meta-analysis. J Int Med Res. 2018;46:3948-58.

6. Losada M Héctor, Muñoz C César, Burgos S Luis, Silva A Jorge. Protocolo de tratamiento y resultados de pancreatitis aguda: Estudio de cohorte. Rev Chil Cir. 2010;62:557-63.

7. Banks PA, Freeman ML. Practice guidelines in acute pancreatitis. Am J Gastroenterol. 2006;101:2379-400.

8. Rappoport J, Fernández J, Valenzuela A, Hernández E. Impacto de la nutrición parenteral total en pancreatitis aguda. Rev Chil Nutr. 1989;17:207-13.

9. McClave SA, Greene LM, Snider HL,
Makk LJ, Cheadle WG, Owens NA, et al. Comparison of the safety of early enteral versus parenteral nutrition in mild acute pancreatitis. JPEN 1997;21:14-20.

10. Zazzo JF. Nutrition des pancréatites aiguès. En: Leverve, Cosnes J, Erny P, Hasselmann M. Traité de Nutrition Artificielle del adulte. París: Editions Mariette Guena, 1998;793-803.

11. Van Felius I, Akkermans L, Bosscha K, Verheem A, Harmsen W, Visser M, et al. Inter digestive small bowel motility and duodenal bacterial over growth in experimental acute pancreatitis. Neurogastroenterol Motil. 2003;15:26727.

12. Song, J, Zhong, Y, Lu, X, Kang, X, Wang, Y, Guo, W, et al. Enteral nutrition provided within 48 hours after admission in severe acute pancreatitis. Medicine (Baltimore). 2018;97: p.e11871. doi: 10.1097/MD.0000000000011871.

13. Wu P, Li L, Sun W. Efficacy comparisons of enteral nutrition and parenteral nutrition in patients with severe acute pancreatitis: a metaanalysis from randomized controlled trials. Biosci Rep. 2018;38(6):BSR20181515. doi: 10.1042/ BSR20181515.

14. Gompertz M, Lara I, Fernández L, Miranda J, Mancilla C, Watkins G, et al. Mortalidad de la pancreatitis aguda: experiencia de 20 años en el Hospital Clínico Universidad de Chile. Rev Med Chile 2013;141:562-7.

15. Sekimoto M, Takada T, Kawarada Y, Hirata K, Mayumi T, Yoshida M, et al. JPN Guidelines for the management of acute pancreatitis: epidemiology, etiology, natural history, and outcome predictors in acute pancreatitis. Journal of HepatoBiliary-PancreaticSurgery 2006;13:10-24.

16. Lankisch P, Apte M, Banks P. Acute pancreatitis The Lancet.
2015;386(9988):85-96. doi: 10.1016/ S0140-6736(14)60649-8

17. Petrov MS, Pylypchuk RD, Uchugina AF. A systematic review on the timing of artificial nutrition in acute pancreatitis. $\mathrm{Br}$ J Nutr. 2009;101:787-93.

18. Yadav D, Lowenfels A. Trends in the epidemiology of the first attack of acute pancreatitis: a systematic review. Pancreas 2006;33:323-30 [Erratum, Pancreas 2007;34:174.

19. Besselink M, van Santvoort H, Boermeester M, Nieuwenhuijs V, van Goor H, Dejong C, et al. Timing and impact of infections in acute pancreatitis. Br J Surg. 2009;96:267-73.

20. Zhao X, Zhu S, Xue G, Li J, Liu Y, Wan $\mathrm{M}$, et al. Early oral refeeding based on hunger in moderate and severe acute pancreatitis: A prospective controlled, randomized clinical trial. Nutrition 2015;31:171-5. doi: 10.1016/j. nut.2014.07.002

21. Al-Omran M, Albalawi Z, Tashkandi M, Al-Ansary L. Enteral versus parenteral nutrition for acute pancreatitis. Cochrane Database Syst Rev. 2010 Jan20;2010(1):CD002837. doi: 10.1002/14651858.CD002837.pub2.

22. McClave S, Martindale R, Vanek V, Mccarthy, M, Roberts P, Taylor B, et al. Guidelines for the provision and assessment of nutrition support therapy in the adult critically ill patient: Society of Critical Care Medicine (SCCM) and American Societyfor Parenteral and Enteral Nutrition (A.S.P.E.N.). JPEN J Parenter Enteral Nutr. 2009;33:277-316.

23. Meier R, Ockenga J, Pertkiewicz M, Pap A, Milinic N, MacFie J, et al. ESPEN guidelines on enteral nutrition: pancreas. Clin Nutr. 2006;25:275-84

24. Jones BJ. Enteral feeding: techniques of administration. Gut 1986;27:47-50. 